\title{
Switching Languages, Switching Palabras (Words): An Electrophysiological Study of Code Switching
}

\author{
Eva M. Moreno,* Kara D. Federmeier,* and Marta Kutas*, $\dagger$ \\ Departments of $*$ Cognitive Science and $\uparrow$ Neurosciences, University of California, San Diego
}

\begin{abstract}
Switching languages has often been associated with a processing cost. In this study, the authors used event-related potentials to compare switches between two languages with withinlanguage lexical switches as bilinguals read for comprehension. Stimuli included English sentences and idioms ending either with the expected English words, their Spanish translations (code switches), or English synonyms (lexical switches). As expected, lexical switches specifically enhanced the N400 response in both context types. Code switches, by contrast, elicited an increased negativity over left fronto-central sites in the regular nonidiomatic sentences $(250-450 \mathrm{~ms})$ and a large posterior positivity $(450-850 \mathrm{~ms})$ in both context types. In addition, both lexical and code switches elicited a late frontal positivity $(650-850 \mathrm{~ms})$ relative to expected completions, especially in idioms. Analysis of the individual response patterns showed correlations with vocabulary skills in English and in Spanish. Overall, the electrophysiological data suggest that for some speakers in some contexts, the processing of a code switch may actually be less costly than the processing of an unexpected within-language item. ๑2002 Elsevier Science (USA)
\end{abstract}

Key Words: code switching; event-related potentials; bilinguals; sentence processing; N400; late positive complex; idioms.

\section{INTRODUCTION}

In bilingual communities, it is not uncommon for speakers to alternate between languages - that is, to "code switch" - in the course of a conversation or even a sentence. A bilingual English-Spanish speaker, for example, might produce a sentence such as "The driver of the speeding car was given a multa." In this example, the use of the Spanish word "multa" instead of the English "ticket" may be due to the fact that the speaker does not recall the English word for multa at that point in the sentence and, knowing that he or she is speaking to another bilingual, substitutes the homologous Spanish word instead. In fact, this "most available word" phenomenon is one of the more common reasons that bilinguals give for code switching: "I tend to use both languages when I feel tired or lazy as an 'easy way out' when I cannot find a word in the language I am speaking', (quoted in Grosjean, 1982). Bilinguals thus may code switch when they cannot find or do not know an

This work was supported by Grants HD22614 and AG08313 to M.K. We thank Cindy Lou Cantu for help with pilot data collection, Nicole Wicha and Lourdes Anllo-Vento for helpful comments and assistance with Spanish translations, and all of the bilingual speakers who participated in this study.

Address correspondence and reprint requests to Marta Kutas, Department of Cognitive Science, UCSD, 9500 Gilman Drive, La Jolla CA 92093-0515. Fax: 858-534-1128. E-mail: kutas@cogsci.ucsd.edu. 
appropriate within-language word. From the point of view of the bilingual speaker, then, a code switch could be regarded as just a change in language form, with the "same" meaning conveyed using a more available word that happens to be from the other language. In this sense, code switching might be comparable to choosing between synonyms within the same language (Sridhar \& Sridhar, 1980).

From the point of view of the comprehender, however, code switches are unexpected and, perhaps, more difficult to process than the corresponding within-language items. Initial studies found, for example, that bilinguals were slower to read languagemixed passages than single-language passages (Macnamara \& Kushnir, 1971). Interference effects from language changes have also been reported on lexical decision latencies in lists of unrelated words. Bilinguals were slower to recognize words in one language when these were immediately preceded by words from another language (the "basic language priming"' effect), suggesting that switching languages influences word recognition processes (Grainger \& Beauvillain, 1988; Grainger \& O'Regan, 1992). Such studies support the view that recognizing and integrating a linguistic code different from that most recently encountered entails a processing cost for the comprehender. This cost, however, is modulated by factors such as the ease with which a switch can be recognized (e.g., easier when the phonetics of the codeswitched word differ from that of the base language [Grosjean, 1995; Li, 1996]), the ecological validity of the switch (e.g., easier in the auditory modality than in the visual modality [Martinez, Sosa, Bates, \& Hernandez, 1998; see also Chan, Chau, \& Hoosain, 1983]), and the constraint of the context in which the switch is embedded (Li, 1996). In fact, some studies suggest that when the uncertainty associated with a code switch is reduced or eliminated (by blocking experimental stimuli or creating more natural contexts for code switching), the cost of the switch is eliminated as well (Amrhein, 1999; Chan et al., 1983). Other studies find, however, that even a perfectly predictable code switch is associated with some processing cost (Attarriba, Kroll, Sholl, \& Rayner, 1996; Martinez et al., 1998; Meuter \& Allport, 1999).

To fully understand the extent and nature of the costs (or perhaps benefits) entailed by a code switch, it is crucial to understand how that switch is treated by the language comprehension system. For example, are the costs incurred at the level of word recognition and lexical/semantic processing or only later in decision-making stages (as suggested by, e.g., Thomas \& Allport, 2000)? Are code switches simply unexpected events at the physical level, or are there associated semantic processing costs as well? Event-related brain potentials (ERPs) provide an excellent dependent measure for beginning to answer questions like these because they can be obtained during natural comprehension, without the need for an additional task, and have the high temporal resolution needed to determine which aspects of processing may be affected by a language switch.

Previous ERP work has shown that the amplitude of a negativity peaking around $400 \mathrm{~ms}$ post-stimulus onset (N400) is highly associated with processing at the level of meaning (Kutas \& Hillyard, 1980b; see also review by Kutas \& Federmeier, 2000). Words that are semantically unexpected or difficult to integrate into a given semantic context elicit increased N400 amplitudes relative to more predictable words. By contrast, unexpected changes in the physical attributes of words (e.g., size, color) and unexpected language events that are nonsemantic in nature (e.g., grammatical violations) are associated with positive-going potentials in the same or slightly later time periods (P300/P600) (Hagoort, Brown \& Groothusen, 1993; Kutas \& Hillyard, 1980a; Münte, Heinze, Matake, Wieringa, \& Johannes, 1998; Osterhout \& Holcomb, 1992). With ERPs, then, one can determine which aspect(s) of processing is affected by some type of unexpected event such as a code switch.

In this study, therefore, we used ERPs to examine how English-Spanish bilinguals 
(of varying proficiency in both languages) process code switches during language comprehension (reading). To our knowledge, this is the first electrophysiological study to look at code switching. Previous work with English monolinguals has shown that unexpected within-language synonyms (items with low cloze probability) elicit increased N400s relative to more expected (higher cloze probability) words in the same contexts. Here, we extend such paradigms to compare responses to (a) expected words from within the base language, (b) unexpected within-language synonyms ("lexical switches"), and (c) unpredictable code switches (translation equivalents of the expected ending) in the same (English) contexts. If code switches create processing difficulties at the level of lexical access and semantic integration, then they should elicit larger N400 responses, similar to lexical switches. By contrast, insofar as a code switch is treated predominantly as a change in form rather than a change in meaning, we would predict that it would elicit a late positivity rather than an N400, although there also may be some brain activity more generally related to switching and its processing demands.

We further aimed to examine the influence of contextual constraint on the processing of unexpected items by embedding lexical and code switches within both moderately constraining normal English sentences (e.g., "The driver of the speeding car was given a ...'”) and highly constraining English idiomatic phrases ("Too many cooks spoil the ...'”). Idiomatic phrases constrain at the lexical level as well as (or even instead of ) the semantic level, and switches of either type (lexical or code) are thus quite unexpected in such contexts. However, given the high constraint of these contexts, if a code switch were to occur, the Spanish word would be more predictable than in the nonidiomatic contexts. The increased predictability afforded by increased contextual constraint might be especially important for processing by individuals who are less fluent in either the base language or the code-switched language. To look at this, we explored individual differences in the responses to the ending types in the two context types as a function of both English and Spanish proficiency.

In sum, with this study we aim to elucidate the nature of the processing that code switching engenders and to compare it to within-language lexical switches. We also examine the interaction of these processes with the nature and strength of the preceding sentence context. Finally, we examine individual differences in the response to the various conditions, making a preliminary examination of the role that language fluency (in both the base and code-switched language) may play in modulating the ease and efficiency with which a bilingual speaker deals with a code-switched message.

\section{METHODS}

\section{Materials}

Materials were 210 sentence contexts in English that ended with three types of target words. Half of the sentence contexts consisted of moderately constraining, normal English sentences ("regular contexts"') used in previous ERP studies (e.g., "The ship disappeared into the thick ...') (Kutas \& Hillyard, 1984). The other half consisted of common idiomatic (and thus highly constrained) sayings ("idiomatic contexts") (e.g., "Too many cooks spoil the ...'). The two types of contexts were matched for length. Across the experiment, all sentences ended with three different types of target words. Expected completions were the highest cloze probability completion ${ }^{1}$ of the regular contexts (e.g., "fog"') and the canonical completion of the idiomatic contexts as given by a proverb dictionary (e.g., "broth"). Lexical

\footnotetext{
${ }^{1}$ The "cloze probability" of a word in a sentence context refers to the proportion of individuals who complete that particular sentence with that particular word when given the sentence contexts off-line (Taylor, 1953).
} 
TABLE 1

Sentence and Target Word Examples Used in the Study

\begin{tabular}{|c|c|c|c|}
\hline & Expected & $\begin{array}{l}\text { Code } \\
\text { switch }\end{array}$ & $\begin{array}{l}\text { Lexical } \\
\text { switch }\end{array}$ \\
\hline \multicolumn{4}{|l|}{ Regular contexts } \\
\hline Each night the campers built a . . & fire & fuego & blaze \\
\hline He put a clean sheet on the ... & bed & cama & mattress \\
\hline The driver of the speeding car was given a & ticket & multa & citation \\
\hline He heard a knock at the . & door & puerta & entrance \\
\hline She put on her high heeled & shoes & zapatos & boots \\
\hline \multicolumn{4}{|l|}{ Idiomatic contexts } \\
\hline Out of sight, out of . . & mind & mente & brain \\
\hline The truth hit me like a ton of . . & bricks & ladrillos & stones \\
\hline A dog is a man's best... & friend & amigo & buddy \\
\hline The grass is always greener on the other & side & lado & lot \\
\hline Ann doesn't know enough to come in out of the & rain & lluvia & drizzle \\
\hline
\end{tabular}

switches were congruent but low-probability completions derived using English synonyms of the expected completions (e.g., "mist" and "bouillon"). Finally, code switches were literal Spanish translations of the expected completions (e.g., "niebla" and "caldo"). Translations into Spanish were done by a native Spanish speaker (E.M.) and confirmed by two other native Spanish speakers. Table 1 gives additional examples of the stimuli.

Sentence-final targets were matched for word length and word frequency across regular and idiomatic contexts for each ending type (English frequency information from Francis \& Kucera, 1982; Spanish frequency information from Juilland and Chang-Rodriguez, 1964). Overall, Spanish endings were longer and less frequent than the expected completions in English. However, lexical switches were matched to code switches for both word length and frequency, as seen in Table 2. Three experimental lists were constructed such that no context and no target were repeated within a list (word frequency and length were controlled within as well as across lists). Each list thus contained 35 stimuli in each constraint by ending type condition. Stimuli were randomized once within each list and then presented in the same order for each participant. Participants were randomly assigned to a list.

\section{Participants}

A total of 37 English-Spanish bilingual speakers (28 females and 9 males) between the ages of 18 and 39 years (mean age 22) were paid for their participation in the study. They were recruited through advertisements on the University of California, San Diego, campus. All were right-handed (as assessed by the Edinburgh inventory (Oldfield, 1971)) and had near-native fluency with and frequent non-classroom use of both languages. Of the 37 subjects, 3 had to be dropped because of their lack of familiarity with the idiomatic contexts (as assessed at the end of the experiment). Results are thus reported for a final set of 34 subjects ( 25 females and 9 males) with mean age 22 years.

Participants' proficiency with each language was assessed in two ways. First, all completed a questionnaire asking them to rate their proficiency with comprehension, speaking, reading, and writing for each language. Self-reports were on a scale of 1 to 7 , where 1 was almost none and 7 was like a native speaker. Average English proficiency (range in parentheses) was: 6.82 (4-7) for comprehension, 6.82 (5-7) for speaking, 6.85 (5-7) for reading, and 6.55 (4-7) for writing. Spanish proficiency was reported to be slightly lower overall: 6.14 (4-7) for comprehension, 5.85 (4-7) for speaking, 5.76 (4-7) for

TABLE 2

Mean Word Frequency and Length across Experimental Conditions

\begin{tabular}{|c|c|c|c|c|c|c|}
\hline & \multicolumn{2}{|c|}{ Expected completion } & \multicolumn{2}{|c|}{ Code switch } & \multicolumn{2}{|c|}{ Lexical switch } \\
\hline & Regular & Idiomatic & Regular & Idiomatic & Regular & Idiomatic \\
\hline Frequency & 100.3 & 97.7 & 43.8 & 46.9 & 43.2 & 42.4 \\
\hline Length & 4.8 & 4.9 & 6.0 & 6.1 & 5.7 & 6.4 \\
\hline
\end{tabular}


reading, and 5.38 (4-7) for writing. In all cases, however, participants believed themselves to have at least "functional" proficiency with all subskills in both languages.

Vocabulary skills in English and Spanish also were assessed using the Boston Naming Vocabulary Test (BNT) (Kaplan, Goodglass, \& Weintraub, 1983). The BNT consists of 60 outline drawings of objects and animals that subjects are asked to name. Here, we asked participants to name the line drawings in both languages (the complete set in one language followed by the complete set in the other, with order counterbalanced across subjects). In English, test items decrease in word frequency and increase in difficulty across the set, from item 1 ("bed") to item 60 ("abacus"). This sequential organization in terms of difficulty does not strictly apply to the Spanish names for these objects, but there is a positive correlation between the degree of difficulty in English and the degree of difficulty in Spanish (Kohnert, Hernandez \& Bates, 1998). Participants correctly named an average of 53 items in English (range 45-60) and 35 items in Spanish (range 13-58). English vocabulary and Spanish vocabulary were uncorrelated $\left(R^{2}=.005\right), F(1,32)=0.17, p=$ n.s. Thus, the simple difference between participants' scores on the English and Spanish versions of the BNT (English-Spanish; "differential score') was used as a rough measure of language dominance. Differential scores ranged from 39 (very English dominant) to -10 (somewhat Spanish dominant).

\section{Procedure}

At the start of a session, volunteers completed a handedness questionnaire (Oldfield, 1971) and a language history questionnaire provided by the Center for Research in Language at the University of California, San Diego. Testing then took place in a single experimental session conducted in a soundproof, electrically shielded chamber. Participants were seated approximately $60 \mathrm{~cm}$ in front of a computer monitor and instructed to read the experimental sentences for comprehension such that they could later answer questions about what they had read. They were informed that they would primarily be reading in English but that they would encounter some Spanish words. The session began with a short practice set designed to acclimate volunteers to the experimental conditions.

Experimental sentences were presented one word at a time, centered in a fixation box in the middle of the screen. Non-sentence-final words were presented for a duration of $200 \mathrm{~ms}$ with a 500-ms stimulus onset asynchrony. Sentence-final target words were presented for a duration of $500 \mathrm{~ms}$. Participants were asked to minimize blinking and horizontal eye movements during sentence presentation. A rest screen followed each sentence, and participants pressed a button to initiate the next trial. Periodic breaks were given throughout the experiment.

After the ERP recording session, participants were given a list of the idiomatic contexts missing their final words. They were asked to complete each context with the word that they believed appropriately completed the idiom. For each, they were also asked to indicate whether in the experimental session they had read the sentence with the ending they expected, a different English word, or a Spanish word. On average, participants were able to correctly (i.e., canonically) complete 76 of the 105 idiomatic contexts ( 3 participants who completed fewer than 45 items correctly were dropped, as mentioned previously). Experimental trials for which participants did not report the canonical (expected) completion were excluded from later analysis. After completion of the experimental session, participants were administered the BNT in both languages.

\section{EEG Recording and Analysis}

Scalp electrical activity was recorded from 26 geodesically spaced tin electrodes embedded in an elastic cap and referenced online to the left mastoid. The head icon in Fig. 1 shows the electrode arrangement (X's), with 10-20 system locations (O's) marked for comparison. Electrodes placed on the outer canthus and infraorbital ridge of each eye were used to monitor blinks and horizontal eye movements. Electrode impedances were kept below $5 \mathrm{~K} \Omega$. The signals were amplified within a bandpass of 0.01 to $100 \mathrm{~Hz}$, continuously digitized at $250 \mathrm{~Hz}$, and stored on hard disk for later analysis.

Data were re-referenced offline to the average of the left and right mastoids. Trials contaminated by eye movements, excessive muscle activity, or amplifier blocking were rejected automatically (using thresholds that were set during visual inspection of the data) before averaging. Fewer than $5 \%$ of trials were lost due to such artifacts. Blinks were corrected via a spatial filter algorithm devised by Dale (1994), and a bandpass filter of 0.1 to $20 \mathrm{~Hz}$ was also applied. ERPs were then computed for epochs extending from $100 \mathrm{~ms}$ before stimulus onset to $920 \mathrm{~ms}$ after stimulus onset. Average ERPs to sentence-final words were obtained for each type of target ending (expected completions, lexical switches, and code switches) after subtraction of the 100-ms prestimulus baseline. 

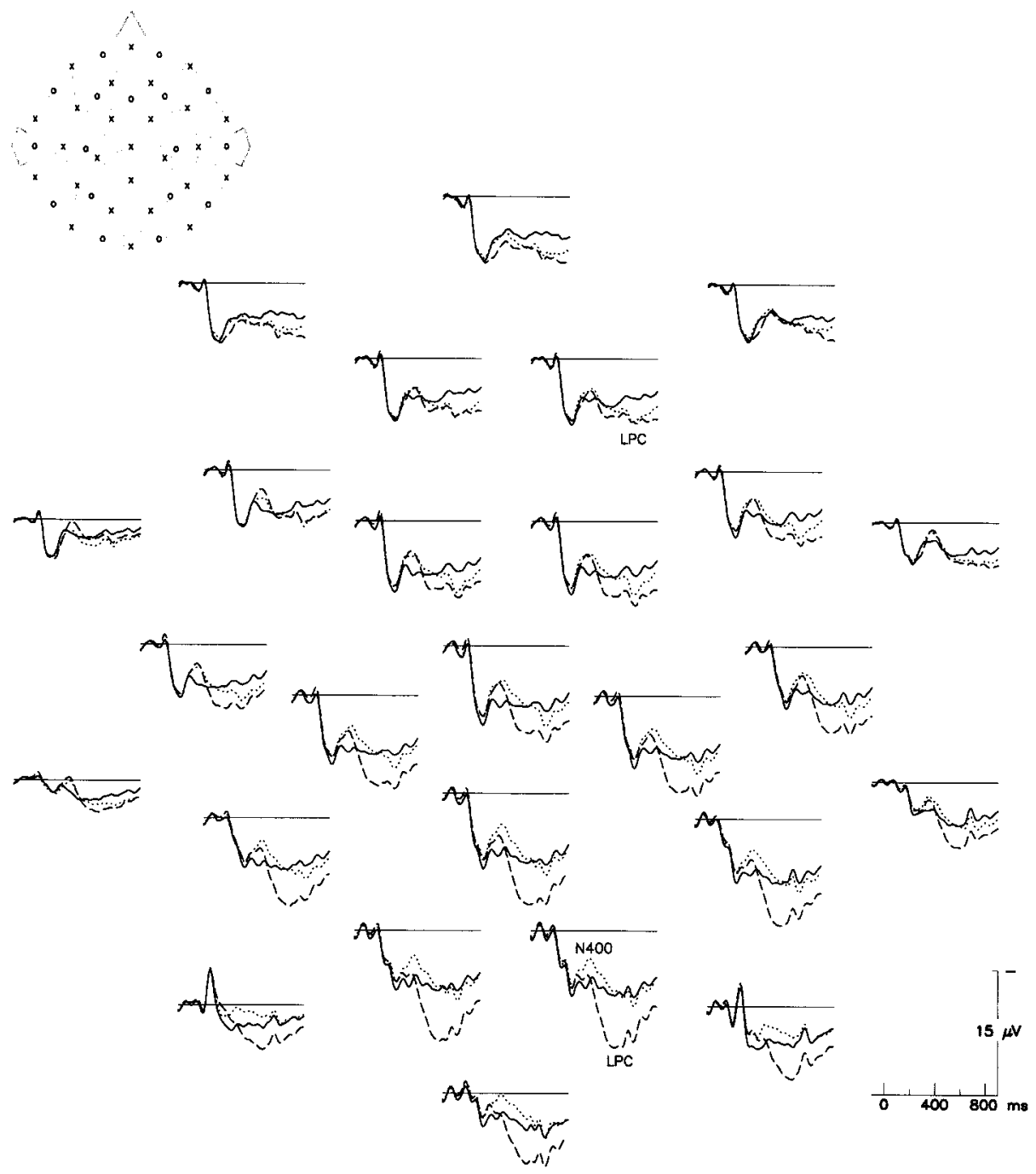

\section{Expected Completion}

Lexical Switch

Code Switch

FIG. 1. Grand average $(N=34)$ ERP waveforms are shown at all 26 electrode sites for the three ending types (expected completions, lexical switches, and code switches) collapsed across context type (regular and idiomatic). The electrode arrangement approximates the layout of the sites on the head, with frontal sites at the top of the figure. The head icon on the upper left of the figure shows the arrangement of the electrodes (X's) with reference to the location of sites in a standard 10-20 system (O's); the 4 midline sites in the geodesic arrangement exactly correspond (from front to back) to $\mathrm{FpZ}, \mathrm{Cz}, \mathrm{Pz}$, and $\mathrm{Oz}$, respectively. Negative is plotted up. Relative to expected completions (solid lines), lexical switches (dotted lines) elicit an enhanced negativity at around $400 \mathrm{~ms}$ (N400) and, beginning around $600 \mathrm{ms,}$ elicit an enhanced positivity over frontal sites. Code switches (dashed lines) also elicit a somewhat enhanced negativity around $400 \mathrm{~ms}$ followed by a large late positive complex (LPC), which is especially pronounced over the back of the head. 


\section{RESULTS}

Figure 1 shows grand average $(N=34)$ ERPs to sentence-final targets (expected completions, lexical switches, and code switches) collapsed across the two types of sentence contexts. Relative to expected completions, lexical switches elicited enhanced negativity over central-posterior sites between 250 and $450 \mathrm{~ms}$ (N400) and, beginning around $600 \mathrm{~ms}$, enhanced positivity over frontal sites (late positive complex [LPC]). Code switches also elicited somewhat enhanced negativity between 250 and $450 \mathrm{~ms}$, followed by enhanced positivity (LPC) over frontal sites. In addition, code switches were characterized by a large posterior positivity (LPC) beginning around $450 \mathrm{~ms}$. These effects varied as a function of the type of context (regular vs idiomatic), as shown in Fig. 2.

\section{Overall Analyses}

Mean amplitudes were measured from 250 to $450 \mathrm{~ms}$ (N400), from 450 to 650 ms (early LPC), and from 650 to $850 \mathrm{~ms}$ (late LPC). These were subjected to an omnibus analysis of variance (ANOVA) on three repeated measures: 2 levels of Constraint (regular vs idiomatic contexts), 3 levels of Ending Type (expected comple-

\section{REGULAR SENTENCES}

Left

Lateral

Prefrontal

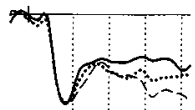

Medial

Frontal

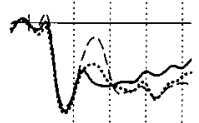

Medial Central

Loteral Occipital

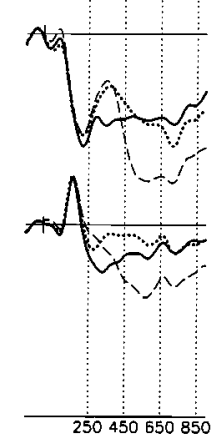

Right
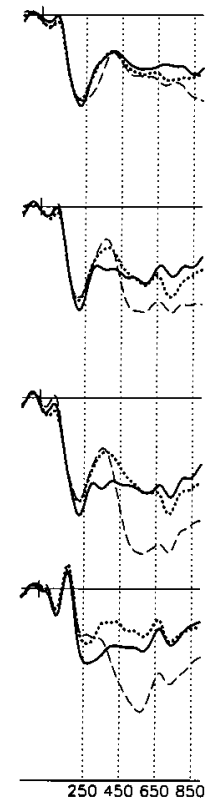

Left
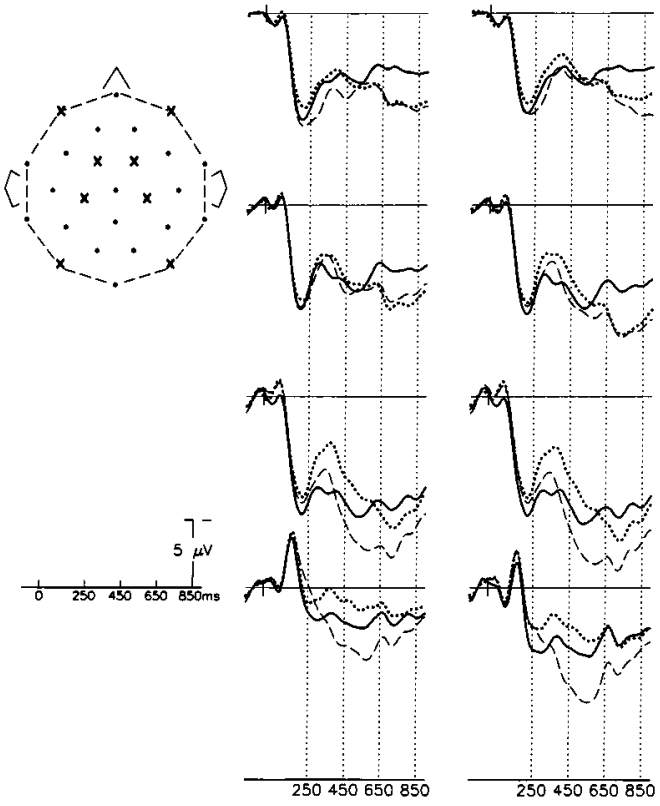

Expected

Lexical switch

Code switch

FIG. 2. Grand average responses to the three ending types are shown at eight representative channels for regular contexts (left) and idiomatic contexts (right). Sites are illustrated with X's on the head icon and include left and right lateral prefrontal (in the 10-20 system, midway between Fp1 and F7 and between Fp2 and F8, respectively), left and right medial frontal (midway between F3/F4 and Cz, respectively), left and right medial central (slightly more posterior and medial than $\mathrm{C} 3$ and $\mathrm{C} 4$, respectively), and left and right lateral occipital (midway between $\mathrm{T} 5$ and $\mathrm{O} 1$ and between $\mathrm{T} 6$ and $\mathrm{O} 2$, respectively). The three time windows used for analyses are delineated by the dotted vertical lines. 
TABLE 3

Overall Results

\begin{tabular}{llccc}
\hline & & $250-450 \mathrm{~ms}$ & $450-650 \mathrm{~ms}$ & $650-850 \mathrm{~ms}$ \\
\hline Constraint & $F(1,33)$ & $14.38^{* *}$ & $13.78^{* *}$ & $13.82^{* *}$ \\
Constraint $\times$ Electrode & $F(25,825)$ & $6.88^{* *}$ & $3.99^{* *}$ & $8.49^{* *}$ \\
Ending Type & $F(2,66)$ & $10.29^{* *}$ & $30.90^{* *}$ & $26.35^{* *}$ \\
Ending Type $\times$ Electrode & $F(50,1650)$ & $8.81^{* *}$ & $20.97^{* *}$ & $12.32^{* *}$ \\
Constraint $\times$ Ending Type & $F(2,66)$ & $4.77^{* *}$ & 1.25 & 2.06 \\
Constraint $\times$ Ending Type $\times$ Electrode & $F(50,1650)$ & $1.93^{*}$ & 1.26 & $2.07^{*}$ \\
\hline
\end{tabular}

$* p<.05$.

$* * p<.01$.

$\# p=0.06$ (n.s).

tions, lexical switches, or code switches), and 26 levels of Electrode. Table 3 gives the results. All $p$ values in this analysis and all subsequent analyses are reported after epsilon correction (Huynh-Felt) for repeated measures with more than 1 degree of freedom.

Both Constraint and Ending Type significantly affected the results in all three time windows. Responses in idiomatic contexts were more positive than responses in regular contexts throughout the epoch. In the 250- to 450-ms time window, responses to expected endings were most positive and responses to lexical switches were most negative. Responses to code switches were then most positive in both the 450- to 650- and 650- to 850-ms time windows. Between 450 and $650 \mathrm{~ms}$, responses to expected exemplars continued to be more positive than responses to lexical switches. This effect then reversed in the 650- to 850-ms time window. Constraint and Ending Type effects interacted in the 250- to 450-ms window, and there was a Constraint by Ending Type by Electrode interaction in the 650- to 850-ms window, suggesting different patterns of response to the three ending types as a function of context type over at least some electrode locations. In the 450- to 650-ms window, the pattern of Ending Type differences did not differ as a function of context. For further analyses in this time window, therefore, the two context types were combined.

\section{Planned Comparisons}

To further characterize the data, we conducted planned comparisons (in the same three time windows) to examine the impact of contextual constraint on the mean amplitude responses to each type of ending and to compare the ending types in each context. In each case, omnibus ANOVAs were conducted on either 2 levels of Constraint or 2 levels of Ending Type and 26 levels of Electrode. A bar graph of these mean amplitude measures (with standard errors) is given in Fig. 3. For space considerations, interactions with electrode are reported only when these were of theoretical significance. ${ }^{2}$ In such cases, significant interactions were followed up by first normalizing the data (McCarthy \& Wood, 1985) and then subjecting the normalized data to a distributional analysis in which the original Electrode factor was subdivided into 2 levels of Hemisphere (left vs right), 2 levels of Laterality (lateral vs medial), and 4 levels of Anteriority (prefrontal, frontal, central, or occipital).

\footnotetext{
${ }^{2}$ That is, we do not report interactions with Electrode when these are predictable and when their presence simply confirms an obvious electrophysiological difference but allows no additional inferences. For example, if one ending type elicits a posterior late positivity while another does not, an interaction will be observed when these conditions are compared since the distribution of the two effects is not the same. Since no other inferences would be made in this case, the interaction would not be reported.
} 


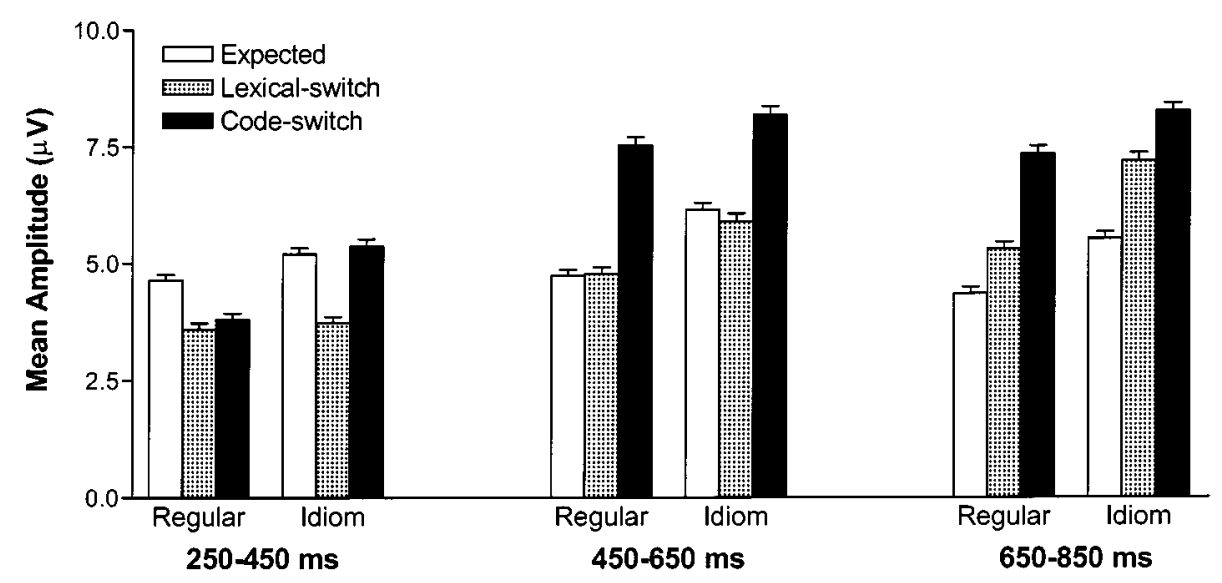

FIG. 3. Bar graph showing mean amplitude measures as a function of context type (regular or idiom) and ending type (expected, lexical switch, or code switch) in the three measurement time windows (250$450,450-650$, and 650-850 ms). Error bars indicate standard errors.

\section{Response to Expected Completions}

Overall, the response to expected completions was more positive $(\sim 1 \mu \mathrm{V})$ in idioms than in regular sentences. The effect was marginal in the 250- to 450 -ms time window, $F(1,33)=3.09, p=.09$, but significant by the 650 - to 850 -ms window, $F(1,33)=9.74, p<.01$, when the response in idiomatic contexts was $5.5 \mu \mathrm{V}$ and the response in regular contexts was $4.3 \mu \mathrm{V}$.

\section{Response to Lexical Switches}

250-450 $\mathrm{ms}$. In both types of contexts, the response to lexical switches was more negative between 250 and $450 \mathrm{~ms}$ than was the response to expected completions (regular: $F(1,33)=10.29, p<.01$; idiomatic: $F(1,33)=4.77, p<.05)$. To compare the N400 effect across contexts, difference waves (response to lexical switch minus response to expected completions) were created, and the mean amplitude difference between 250 and $450 \mathrm{~ms}$ was measured for each context type. Figure 4 shows these difference waves overlapped by context type. N400 effects to lexical switches did not differ as a function of context in either amplitude $(-0.52 \mu \mathrm{V}$ in regular and $-0.73 \mu \mathrm{V}$ in idiomatic contexts), $F(1,33)=1.03, p=$ n.s., or distribution, $F(25$, $825)=1.40, p=$ n.s.

450-650 ms. Responses to lexical switches in this time window did not differ from those to expected completions, $F(1,33)=0.02, p=$ n.s.

650-850 ms. Responses to lexical switches were more positive than responses to expected completions in this time window for both context types (regular: $F(1$, $33)=4.81, p<.05$; idiomatic: $F(1,33)=15.85, p<.01)$. This effect is especially prominent over more frontal electrode sites, as can be seen in Fig. 2. When compared directly, responses were found to be more positive for lexical switches in idioms (7.2 $\mu \mathrm{V})$ than in regular sentences $(5.3 \mu \mathrm{V}), F(1,33)=12.34, p<.01$.

\section{Response to Code Switches}

250-450 $\mathrm{ms}$. Like the response to lexical switches, the response to code switches in regular contexts was more negative than the response to expected completions, $F(1,33)=6.24, p<.05$. However, responses to code switches in idiomatic contexts 
Lexical switches - Expected

Left

Loteral

Prefrontal
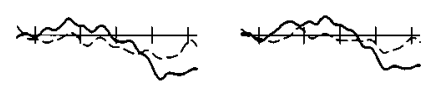

Right

Medial
Frontal
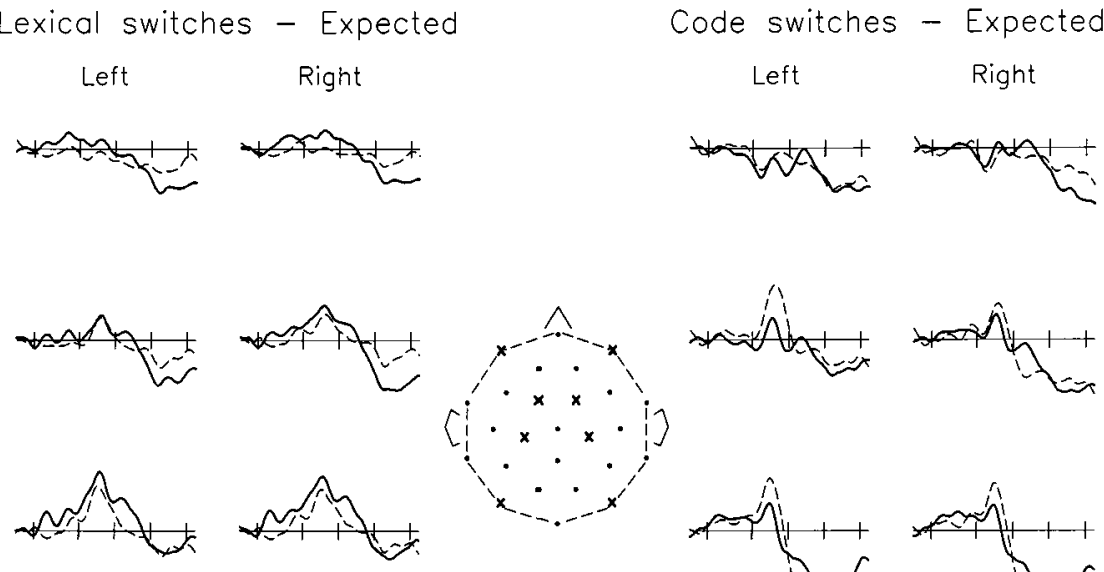

Medial

Central
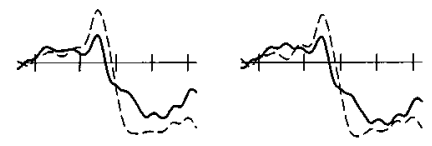

Lateral

Occipitol

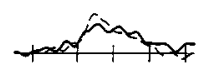

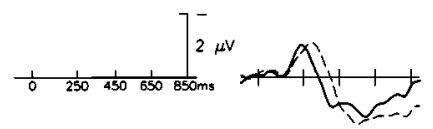

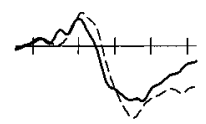

Idioms

Regular sentences

FIG. 4. Difference waves (switch ERP minus expected completion ERP) for lexical switches (left) and code switches (right) in the two context types. N400 effects to lexical switches were similar in both context types, and lexical switches also elicited an increased late frontal positivity, particularly in idiomatic contexts (solid line). Between 250 and $450 \mathrm{~ms}$, code switches in regular contexts elicited a left frontally distributed negativity (dashed line). Beginning around $450 \mathrm{~ms}$, code switches in both context types elicited a large posterior positivity, which around $650 \mathrm{~ms}$ overlaps with a frontal positivity similar to that seen for lexical switches.

did not differ from those to expected completions in this time window, $F(1,33)=$ $0.12, p=$ n.s. $^{3}$ Again, difference waves (code switch minus expected completion) were computed and compared across the two contexts (Fig. 4), confirming a larger effect of code switching in regular contexts than in idiomatic contexts $(-0.42 \mu \mathrm{V}$ in regular and $0.08 \mu \mathrm{V}$ in idiomatic contexts); $F(1,33)=3.96, p=.05$; this effect interacted with Electrode, $F(25,825)=3.00, p=.01$. A distributional analysis on the difference waves revealed a Constraint by Hemisphere interaction, $F(1,33)=$ $9.52, p<.01$, that was modulated by a Constraint by Hemisphere by Laterality interaction, $F(1,33)=5.02, p<.05$, and a marginal Constraint by Hemisphere by Laterality by Anteriority interaction, $F(3,99)=2.53, p=.06$. Opposite to the pattern in idiomatic contexts, code switch effects in regular sentences were larger over the left side than the right side of the scalp, especially over lateral anterior sites. This distributional pattern is not that typically observed for N400 effects, which are largest over right medio-central sites.

450-650 ms. In this time window, the response to code switches was more positive than the response to expected completions, $F(1,33)=34.01, p<.01$, especially over posterior electrode sites.

650-850 ms. Again in this time window, the response to code switches was more positive than the response to expected completions in both types of context (regular: $F(1,33)=28.67, p<.01$; idiomatic: $F(1,33)=38.85, p<.01)$. As was true for

${ }^{3}$ No significant differences emerge for this comparison even when analyses are restricted to the first half of the time window (250-350 ms) when there is little overlap with the subsequent LPC. 
lexical switches, the positivity was approximately $1 \mu \mathrm{V}$ larger for code switches in idioms $(8.2 \mu \mathrm{V})$ than for code switches in regular sentences $(7.3 \mu \mathrm{V}), F(1,33)=$ $4.19, p=.05$.

\section{Comparison of Lexical Switches and Code Switches}

250-450 ms. Analyses on the difference waves revealed greater negativity for lexical switches than for code switches when these were embedded in idiomatic contexts, $F(1,33)=16.20, p<.01$. The interaction with Electrode was also significant, suggesting a possible distributional difference between these effects, $F(25,825)=$ $3.84, p<.05$. Distributional analyses showed a significant Ending Type by Anteriority interaction, $F(3,99=6.39, p<.05$, modulated by a significant Ending Type by Hemisphere by Anteriority interaction, $F(3,99)=7.31, p<.01$, and a marginal Ending Type by Laterality by Anteriority interaction, $F(3,99)=2.91, p=.06$. Lexical switches in idiomatic contexts elicited a distribution typical of an N400 effect, with the most negative responses over central and posterior sites, bigger medially than laterally and bigger over right than left scalp sites. Responses to code switches in idiomatic contexts showed a similar pattern, but only over more frontal electrode sites (perhaps in part because of overlap with the posterior positivity that onsets toward the end of this time window).

Whereas the effect size for lexical and code switches differs in idioms, it does not differ in regular sentences, $F(1,33)=0.46, p=$ n.s. However, the interaction with Electrode was again significant, $F(25,825)=8.97, p<.01$, and follow-up analyses indicate a different distribution of the negative effect elicited by lexical switches and code switches in regular contexts. The distribution of the effect to lexical switches was the same as that observed for these items in idiomatic contexts and thus canonical of an N400 effect. The response to code switches, by contrast, was largest more frontally and had a striking left lateral skew (see Fig. 5). This response, thus, was

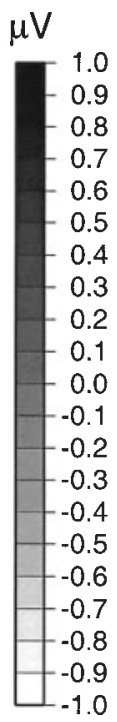

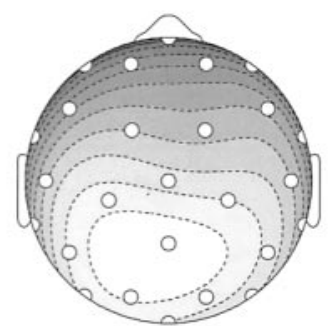

Lexical switch

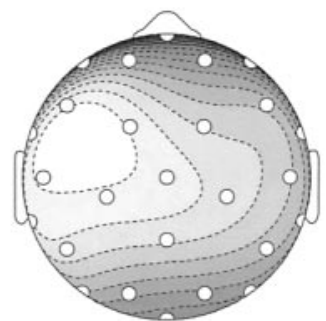

Code switch

FIG. 5. Topographic maps showing the voltage distribution (350-450 ms) of the negative effect (switch ERP minus expected ERP) observed for lexical switches (left) and code switches (right) in regular contexts. Relative to expected completions, both types of switches elicited increased negativity in this time window. However, the distribution of the effect for lexical switches is canonical of N400 effects, with a medial posterior focus. The effect for code switches, by contrast, is more frontal, with a striking left lateral skew. 
uncharacteristic in distribution of an N400; instead, it might be akin to a LAN (left anterior negativity) effect (e.g., Neville, Nicol, Barss, Forster \& Garrett 1991; Friederici, Pfeifer \& Hahne, 1993; Münte, Heinze, \& Mangun, 1993)

$450-650 \mathrm{~ms}$. In this time window, the response to code switches was more positive than the response to lexical switches (which did not differ from the response to expected completions), $F(1,33)=55.32, p<.01$.

$650-850 \mathrm{~ms}$. The response to code switches was more positive than the response to lexical switches in both contexts (regular: $F(1,33)=15.72, p<.01$; idiomatic: $F(1,33)=6.41, p<.05$. Distributional analyses (collapsed across context types) reveal a significant Ending Type by Anteriority interaction, $F(3,99)=20.42, p<$ .01 , modulated by an Ending Type by Hemisphere by Anteriority interaction, $F(3$, $99)=3.55, p<.05$, and an Ending Type by Laterality by Anteriority interaction, $F(3,99)=3.95, p<.05$. In this time window, responses to the two types of switches were quite similar over the front of the head; it is over the back of the head that code switches elicited greater positivity than lexical switches (more so over left than right hemisphere and more so over lateral than medial sites). It thus seems that this ending type difference is the continuation of the posterior positivity, which began in the 450to 650 -ms time window, and that the frontal positivity in this time window is similar for the two switch types.

\section{Summary of the Group Results}

Relative to expected completions, lexical switches elicited larger N400s in both types of context. In addition, especially in idiomatic contexts, these within-language switches were associated with a late $(650-850 \mathrm{~ms})$, primarily frontal positivity. By contrast, code switches elicited a large posterior positivity in both context types. This positivity began in the 450 - to 650 -ms time window and continued into the 650 - to 850-ms time window, where it overlapped with a frontal positivity similar to that observed for lexical switches. In addition, code switches in regular sentence contexts were associated with negativity with a left, frontally skewed distribution (LAN?) between 250 and $450 \mathrm{~ms}$.

\section{Individual Differences}

To explore the effects of language proficiency and dominance on the effects just described, we performed regression analyses to see whether N400 responses (to lexical switches) and/or LPC responses (to code switches) were predicted by individuals' scores on the English or Spanish versions of the BNT or by the difference between their performances on the two (their relative language dominance). Figure 6 shows these regression analyses.

\section{N400 (Lexical Switches)}

Differential (English minus Spanish) score on the BNT predicted N400 effect sizes to lexical switches when these were embedded in regular sentences $\left(R^{2}=.11\right)$, $F(1,32)=4.16, p=.05$. More English-dominant individuals had larger effect sizes (mean amplitude difference in the 250- to 450-ms time window between the response to lexical switches and the response to expected completions) than did more balanced (or slightly Spanish-dominant) individuals. ${ }^{4}$ The more English-dominant pattern of

\footnotetext{
${ }^{4}$ Spanish vocabulary scores primarily carried the difference between the more English-dominant bilinguals and the more balanced bilinguals, although a few speakers were Spanish dominant and thus had lower than average English vocabulary scores.
} 


\section{Boston Naming Test (BNT) Score correlations}

\section{Lexical switch $\mathrm{N} 400$}

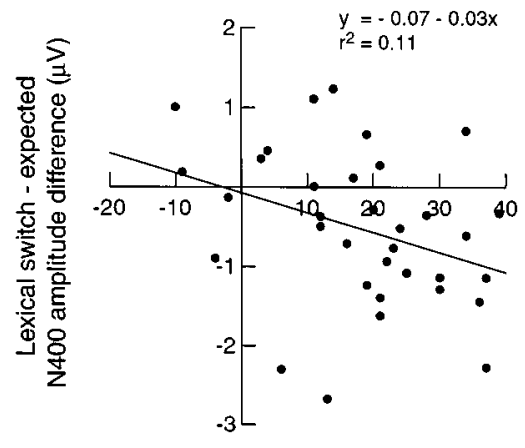

English - Spanish BNT difference

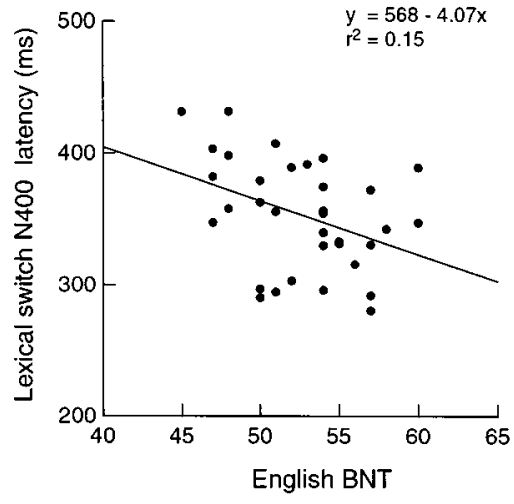

Code switch late positive component (LPC)
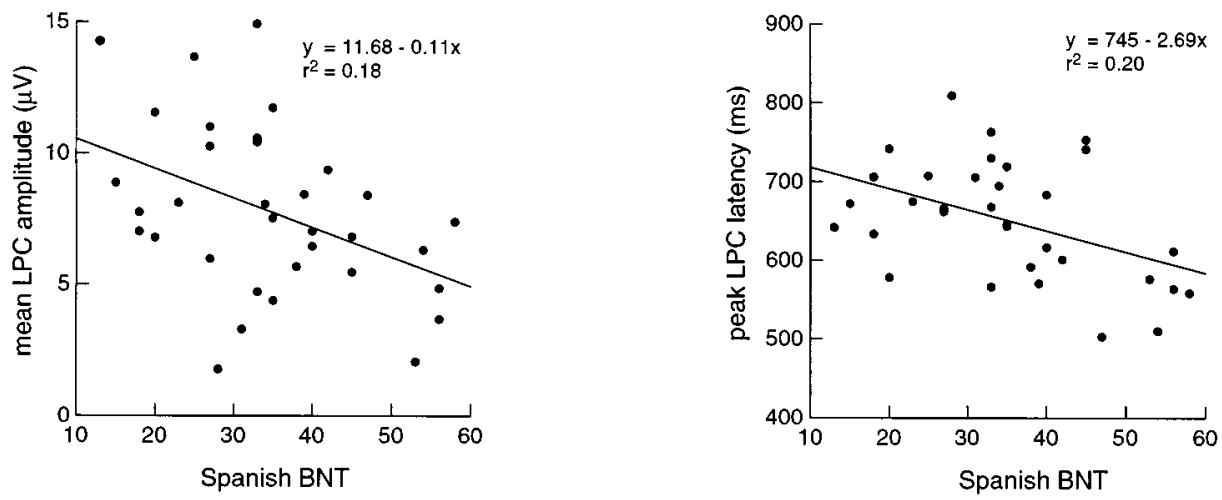

FIG. 6. Plots showing the correlation of individual participants' ERP measures with their Boston Naming Test (BNT) scores. Both the N400 response to lexical switches (top) and the LPC response to code switches (bottom) varied with vocabulary skills. Individuals more dominant in English tended to elicit larger N400 effects to lexical switches (top left), and increased English vocabulary was also predictive of earlier peak N400 responses (top right). Increased Spanish vocabulary was predictive of both smaller (bottom left) and earlier (bottom right) LPC responses to code switches.

N400 responses corresponds to that typically observed for English monolinguals (e.g., Kutas \& Hillyard, 1984). Neither English nor Spanish scores alone predicted this effect, and there was no correlation between N400 effect size for lexical switches in idiomatic contexts and any of the vocabulary measures.

In addition, peak latency of the N400 response to lexical switches in both types of context was predicted by participants' scores on the English version of the BNT. This was true when measures were taken over all channels $\left(R^{2}=.15\right), F(1,32)=$ $5.46, p<.05$, or at a single representative channel $(\mathrm{MiCe})\left(R^{2}=.13\right), F(1,32)=$ 
4.95, $p<.05$. Higher English scores were predictive of earlier N400 peak latencies. Neither Spanish nor differential scores were predictive.

\section{Posterior LPC (Code Switches)}

Both the amplitude and the peak latency of the posterior late positivity elicited by code switches were predicted by participants' scores on the Spanish version of the BNT and, less strongly, by their differential scores. Across all channels between 450 and $850 \mathrm{~ms}$, higher scores on the Spanish version of the BNT (and smaller differential scores, indicative of less English dominance) predicted LPC responses that peaked earlier (Spanish score: $R^{2}=.20, F(1,32)=7.93, p<.01$; differential score: $R^{2}=$ $.14, F(1,32)=5.06, p<.05)$ and were smaller in mean amplitude (Spanish score: $R^{2}=.18, F(1,32)=7.08, p=.01$; differential score: $R^{2}=.16 ; F(1,32)=6.39$, $p<.05)$. English scores were not predictive.

\section{Summary of the Regression Analyses}

Increased proficiency with English predicted earlier N400 responses to lexical switches regardless of context. In addition, those who were relatively more dominant in English showed greater differentiation (in N400 amplitude) between expected items and lexical switches within regular sentences. Increased Spanish proficiency, in turn, predicted earlier LPC responses to code switches and less differentiation of code-switched from non-code-switched items (in LPC amplitude).

\section{DISCUSSION}

In this experiment, we used ERPs to elucidate the nature of the processing engendered when a bilingual comprehender encounters an unexpected switch from one language to another. To that end, we compared the response to such unexpected codeswitches-here, into Spanish-to the response to equally unexpected (and equally comprehensible) lexical items within the base language, English. We also explored the influence of both contextual constraint and an individual bilingual's proficiency with their two languages on these processes.

Unexpected lexical items from within the base language set, termed lexical switches in this experiment, elicited the pattern of ERP responses predicted from previous studies using monolingual speakers (e.g., Gunter, Friederici, \& Schriefers 2000; Kutas \& Hillyard, 1980b, 1984). These low cloze probability items (which, nonetheless, were semantically quite similar to the expected completion) were associated with greater negativity between 250 and $450 \mathrm{~ms}$ (N400) than were the expected completions of the sentences, suggesting that they are more difficult to integrate with the sentence context information. This was true whether the context was a highly constraining idiom (known to the comprehender) or a less constraining regular English sentence. Similar to previous studies, then, we find that the amplitude of the N400 response is affected by the predictability of the lexical item in its context, independent of the constraint of the context itself (Kutas \& Hillyard, 1984). Contextual constraint did, however, affect the size of the positivity to the expected completions, which was larger in the more constraining idiomatic contexts.

We found individual differences in the latency of the N400 to lexical switches as a function of the comprehender's English proficiency (as indexed by a vocabulary measure). Those with less extensive English vocabularies elicited N400s with later peak latencies. This is consistent with Weber-Fox and Neville's (1996) finding of later N400 peak latencies in second-language speakers who had learned the lan- 
guage later in life and had fewer years of experience with it. It thus seems that semantic processing (in particular, those aspects of semantic processing that are reflected in the scalp-recorded N400) operates with a somewhat slower time course in less fluent language comprehenders. In addition, we found that those individuals who were relatively more dominant in English elicited larger N400 effects for lexical switches in regular sentence contexts. More English-dominant individuals thus seem better able to make use of the contextual information in English to differentiate between the ending that best fits the context and a semantically similar-but lexically less preferred-English item. Such individual differences are not apparent in the idiomatic contexts, presumably because all speakers can differentiate effectively when constraint is very strong and affords clear lexical as well as semantic expectations.

In contrast to the pattern observed for lexical switches, code switches were characterized by a large posterior LPC between about 450 and 850 ms post-stimulus onset. Such positivities have been observed in numerous prior studies, which vary widely in stimulus type and task demands (for reviews, see Donchin \& Coles, 1988; Johnson, 1986; Picton, 1992). In general, they are associated with the processing of an unexpected or improbable task-relevant event (e.g., Donchin, 1981; McCallum, Farmer, \& Pocock, 1984). Such events need not be linguistic in nature, although some types of unexpected language events (e.g., certain types of grammatical violations) also elicit similar late positivities (e.g., Coulson, King, \& Kutas, 1998; Münte et al., 1998). In this experiment, words in Spanish were indeed improbable events (as they typically would be in everyday bilingual language processing when English is being used as the base language), constituting a third of the sentence endings. Here, they also were not particularly ecologically valid since they occurred in written text, whereas most code switching occurs in spoken language, and they were from English into Spanish, whereas bilingual speakers in the local community are more likely to code switch from Spanish into English. It is possible, therefore, that the size of this positivity would be reduced by making the code switch a more probable event or by increasing the ecological validity of the switch.

Indeed, we found variation in the size of the LPC, and its peak latency, as a function of speakers' proficiency with Spanish. Those more proficient with Spanish seemed to "notice" the code switch earlier (earlier peak responses) and, furthermore, seemed to find the switch less unexpected and/or less difficult to process (requiring less "context updating"' [Donchin, \& Coles, 1998]), reflected in a smaller amplitude LPC response. Perhaps these individuals found code switching easier because Spanish lexical items are more frequently encountered and better known by these individuals; it is also possible that these speakers are more likely to code switch in their everyday language processing. ${ }^{5}$ Regardless of the specific reasons for the individual differences, however, these results are consistent with findings from behavioral studies suggesting that the "cost" of switching may be modulated by factors affecting how unexpected the switch is for any given individual comprehender.

Code switches thus elicit a qualitatively different ERP response pattern from lexical switches. Unexpected within-language synonyms elicited increased N400 responses in both context types, suggesting that they are more difficult to integrate semantically with the sentence context than are the expected lexical items. By contrast, unpredictable code switches did not elicit increased negativity in idiomatic contexts at all and elicited a negativity that differed in its distributional characteristics from an N400 when embedded in regular contexts. Thus, even under conditions where code switches are less frequent than within-language completions and where

${ }^{5}$ Individuals more proficient in Spanish were no more likely to report frequent code switching in everyday life (on our language questionnaire) than were those less proficient. 
code switching is not particularly ecologically valid, processing a translation equivalent of the expected ending seemed to engender less of a lexical-semantic processing cost than did an unexpected within-language synonym. Along these lines, Kolers and Gonzalez (1980) found that interlingual synonyms (translations) were as beneficial as aids to recall as were exact repetitions, whereas intralingual synonyms (lexical switches) were less effective-suggesting, in their terms, stronger "bonds" between translation equivalents than between synonyms. It may be that small differences in the meaning or use of within-language synonyms are brought out with language experience, resulting in a greater differentiation between similar within-language words than between translation equivalents, which do not compete in the same way. Thus, in our study, it may be that the lexical switches were more semantically distant from the expected completion than were the code switches, resulting in a greater semantic integration difficulty and a concomitantly larger N400 response. The structure, flexibility, and cross-language overlap (or lack thereof) of lexical, semantic, and conceptual levels of representation in bilinguals has long been a controversial and important issue (see, e.g., reviews by Grosjean, 1998, and Pavlenko, 1999). Our electrophysiological results may be taken to suggest that, at least under some circumstances, the semantic information accessed by a word in one language can be fairly similar to that of the "equivalent" word in another language, at least in its processing consequences for sentence comprehension. Thus, even though a code switch may be a surprising event, it need not be more difficult to process at a semantic level. This conclusion clearly does not accord well with any theory in which the architecture of the bilingual language system entails a significant cost to accessing and/or semantically integrating a code-switched item.

Instead of eliciting increased N400 responses, code switches (but not lexical switches) elicited an LPC, suggesting that they may be treated more like unexpected events at a physical level (e.g., Kutas \& Hillyard, 1980a; Osterhout, Bersick, \& McLaughlin, 1997), thereby requiring more resources for stimulus evaluation and memory updating (Brookhuis et al., 1981; Donchin \& Coles, 1998; Kutas, McCarthy \& Donchin, 1997; Sommer, Leufhold, \& Mott, 1998). This supports the hypothesis that the costs associated with processing a code switch may arise predominantly at decision-related stages of processing (e.g., Thomas \& Allport, 2000). While the precise nature of the costs associated with code switching - and the factors that alleviate such costs - remain to be elucidated in full, the results of this experiment clearly show that the language processing system does not regard all types of what might have been classified as "lexically unexpected items" as equivalent.

While we found clear differences in the response to lexical and code switches, we also observed a similar, bilateral frontal positivity in response to both. This positivity developed late in the epoch-between about 650 and $850 \mathrm{~ms}$ - and was larger in response to switches (of either kind) in idiomatic contexts than in regular contexts. Late, frontally distributed effects of this kind have been reported previously in the ERP literature and have been associated with processes related to explicit recall/ recognition (Paller \& Kutas, 1992; Ranganath \& Paller, 1999; Rugg, Allan, \& Birch, 2000), especially of specific, source-related information (Senkfor \& Van Petten, 1998; Van Petten, Senkfor, \& Newberg, 2000). Perhaps, then, when unexpected "switched" items were encountered, participants tended to explicitly bring to mind the expected lexical item, especially in idiomatic contexts where that expected item is so formulaic. Alternatively, it is possible that this potential reflects executive activity generally related to "switching" of any type. While one cannot use the scalp distribution of an ERP effect to directly infer the location of its neural sources, the frontal distribution of this response is at least consistent with a large literature implicating an important role for frontal areas in switching between stimuli and/or tasks (Gershberg \& Shimamura, 1995; Sohn, Ursu, Anderson, Stenger \& Carter, 2000), including 
language switches (Fabbro, Skrap, \& Aglioti, 2000; Hernandez, Martinez, \& Kohnert, 2000; Price, Green, \& Von Studnitz, 1999).

Finally, we found that code switches embedded in regular contexts (although not in idioms) also elicited increased negativity between 250 and $450 \mathrm{~ms}$, with a left frontally skewed distribution (Fig. 5). ${ }^{6}$ Left anterior negativities (LAN effects) have been described previously (e.g., Friederici et al., 1993; Gunter, Stowe \& Mulder, 1997; Münte et al., 1993) and associated with, among other things, syntactic processes, especially those that tax working memory (King \& Kutas, 1995; Kluender \& Kutas, 1993). The finding of a component of this type in response to code switchesand only in regular contexts - was unexpected. Code-switched words in regular and idiomatic contexts were generally matched for part of speech, so we would not expect to find differences based on lexical or morphological aspects of the Spanish words in these two conditions. If this effect is a LAN, then one might speculate that it arises because the syntactic integration of the Spanish word into the English context engenders a working memory load since morphological cues attesting to co-reference and agreement are likely more difficult to process and integrate across languages. Such processes might then be minimized in idiomatic contexts because of their highly lexicalized nature. Clearly, however, further work is needed to understand the basis of this effect and its interaction with contextual constraint.

In summary, we find that the costs associated with processing an unexpected lexical item in a sentence context vary depending on whether that lexical item comes from within the base language or is a word "code switched" from another language. Substituting a less expected within-language synonym for the lexical item predicted by the context seems to make lexical/semantic processing more difficult. If, instead, the expected lexical item is replaced with a cross-language translation equivalent, the processing costs seem to arise at other, perhaps more decision-related, stages of processing. Code switches thus do indeed seem to be treated more as a change in form than as a change in meaning. The extent of the cost engendered by a code switch (as well as a lexical switch) seems to vary with language fluency and likely also will vary as a function of factors such as the probability and ecological validity of the switch. Much further research is clearly needed to uncover how the processing of a code-switched item unfolds in neural and psychological terms and what factors make code switching easier or harder for individual speakers. This initial electrophysiological study, however, clearly indicates that code switches are processed differently from lexical switches and suggests further that, at least for some speakers in some contexts, processing a code switch may actually be less costly at some processing stages than processing a within-language synonym of an expected word.

\section{REFERENCES}

Altarriba, J., Kroll, J. F., Sholl, A., \& Rayner, K. (1996). The influence of lexical and conceptual constraints on reading mixed-language sentences: Evidence from eye fixations and naming times. $\mathrm{Mem}$ ory and Cognition, 24, 477-492.

\footnotetext{
${ }^{6}$ While component overlap can skew the distribution of a component of interest, there are several reasons to believe that the observed negativity to code switches in regular sentences is not just an N400 whose distribution has been obscured by the overlap of the LPC. First, the distribution is characterized not only by lack of activity at locations where N400s are typically prominent (e.g., medial posterior sites-where, indeed, component overlap could be an issue) but also by the presence of negativity at sites where N400 activity is generally fairly minimal (e.g., lateral frontal sites). Second, the distribution of the effect remains distinct from an N400 distribution even in the earlier part of the time window where component overlap is less likely to play a significant role. Finally, inspection of the individual subject data reveals that those manifesting the most prominent left frontal negativities are actually less likely to simultaneously elicit significant LPC activity.
} 
Amrhein, P. C. (1999). On the functional equivalence of monolinguals and bilinguals in "monolingual", mode: The bilingual anticipation effect in picture-word processing. Psychological Science, 10, 230236.

Brookhuis, K. A., Mulder, G., Mulder, L. J., Gloerich, A. B., van Dellen, H. J., van der Meere, J. J., \& Ellermann, H. (1981). Late positive components and stimulus evaluation time. Biological Psychology, 13, 107-123.

Chan, M-C., Chau, H. L., \& Hoosain, R. (1983). Input/output switch in bilingual code switching. Journal of Psycholinguistic Research, 12, 407-416.

Coulson, S., King, J. W., \& Kutas, M. (1998). Expect the unexpected: Event-related brain response to morphosyntactic violations. Language and Cognitive Processes, 13(1), 21-58.

Dale, A. M. (1994). Source localization and spatial discriminant analysis of event-related potentials: Linear approaches. Unpublished doctoral dissertation, University of California, San Diego.

Donchin, E. (1981). Surprise!. . . Surprise? Psychophysiology, 18, 493-513.

Donchin, E., \& Coles, M. G. (1988). Is the P300 component a manifestation of context updating? Behavioral and Brain Sciences, 11, 357-427.

Fabbro, F., Skrap, M., \& Aglioti, S. (2000). Pathological switching between languages after frontal lesions in a bilingual patient. Journal of Neurology and Neurosurgical Psychiatry, 68, 650-652.

Francis, W. N., \& Kucera, H. (1982). Frequency analysis of English usage. Boston: Houghton Mifflin.

Friederici, A. D., Pfeifer, E., \& Hahne, A. (1993). Event-related brain potentials during natural speech processing: Effects of semantic, morphological, and syntactic violations. Cognitive Brain Research, 1(3), 183-192.

Gershberg, F. B., \& Shimamura, A. P. (1995). Impaired use of organizational strategies in free recall following frontal lobe damage. Neuropsychologia, 33, 1305-1333.

Grainger, J., \& Beauvillain, C. (1988). Associative priming in bilinguals: Some limits of interlingual facilitation effects. Canadian Journal of Psychology, 42, 261-273.

Grainger, J., \& O'Regan, J. K. (1992). A psychophysical investigation of language priming effects in two English-French bilinguals. European Journal of Cognitive Psychology, 4, 323-339.

Grosjean, F. (1982). An introduction to bilingualism. Cambridge, MA: Harvard Univ. Press.

Grosjean, F. (1995). A psycholinguistic approach to code-switching: The recognition of guest words by bilinguals. In L. Milroy \& P. Muysken (Eds.), One speaker, two languages. Cross-disciplinary perspectives on code-switching. Cambridge, UK: Cambridge Univ. Press.

Grosjean, F. (1998). Studying bilinguals: Methodological and conceptual issues. Bilingualism: Language and Cognition, 1(2), 131-149.

Gunter, T. C., Friederici, A. D., \& Schriefers, H. (2000). Syntactic gender and semantic expectancy: ERPs reveal early autonomy and late interaction. Journal of Cognitive Neuroscience, 12, 556-568.

Gunter, T. C., Stowe, L. A., \& Mulder, G. (1997). When syntax meets semantics. Psychophysiology, 34, 660-676.

Hagoort, P., Brown, C., \& Groothusen, J. (1993). The syntactic positive shift (SPS) as an ERP measure of syntactic processing. Language and Cognitive Processes, 8, 439-483.

Hernandez, A., Martinez, A., \& Kohnert, K. (2000). In search of the language switch: An fMRI study of picture naming in Spanish-English bilinguals. Brain and Language, 73, 421-431.

Johnson, R. (1986). A triarchic model of P300 amplitude. Psychophysiology, 23, 367-384.

Juilland, A., \& Chang-Rodriguez, E. (1964). Frequency dictionary of Spanish words. The Hague, Netherlands: Mouton.

Kaplan, E., Goodglass, H., \& Weintraub, S. (1983). Boston Naming Test. Philadelphia: Lea \& Febiger.

King, J. W., \& Kutas, M. (1995). Who did what and when? Using word- and clause-level ERPs to monitor working memory usage in reading. Journal of Cognitive Neuroscience, 7, 376-395.

Kluender, R., \& Kutas, M. (1993). Bridging the gap: Evidence from ERPs on the processing of unbounded dependencies. Journal of Cognitive Neuroscience, 5, 196-214.

Kohnert, K. J., Hernandez, A. E., \& Bates, E. (1998). Bilingual performance on the Boston Naming Test: Preliminary norms in Spanish and English. Brain and Language, 65, 422-440.

Kolers, P. A., \& E. Gonzalez. (1980). Memory for words, synonyms, and translations. Journal of Experimental Psychology: Human Learning and Memory, 6, 53-65.

Kutas, M., \& Federmeier, K. D. (2000). Electrophysiology reveals semantic memory use during language comprehension. Trends in Cognitive Science, 4, 463-470. 
Kutas, M., \& Hillyard, S. A. (1980a). Event-related potentials to semantically inappropriate and surprisingly large words. Biological Psychology, 11, 99-116.

Kutas, M., \& Hillyard, S. A. (1980b). Reading senseless sentences: Brain potentials reflect semantic incongruity. Science, 207, 203-205.

Kutas, M., \& Hillyard, S. A. (1984). Brain potentials during reading reflect word expectancy and semantic association. Nature, 307, 161-163.

Kutas, M., McCarthy, G., \& Donchin, E. (1977). Augmenting mental chronometry: The P300 as a measure of stimulus evaluation time. Science, 197, 792-795.

Li, P. (1996). Spoken word recognition of code-switched words by Chinese-English bilinguals. Journal of Memory and Language, 35, 757-774.

Macnamara, J., \& Kushnir, S. L. (1971). Linguistic independence of bilinguals: The input switch. Journal of Verbal Learning and Verbal Behavior, 10, 480-487.

Martinez, E., Sosa, K., Bates, E., \& Hernandez, A. (1998). Code-switching: When will priming "cross over'? Technical Report 9804, Center for Research in Language, University of California, San Diego.

McCallum, W. C., Farmer, S. F., \& Pocock, P. V. (1984). The effects of physical and semantic incongruities of auditory event-related potentials. Electroencephalography \& Clinical Neurophysiology: Evoked Potentials, 59, 477-488.

McCarthy, G., \& Wood, C. C. (1985). Scalp distributions of event-related potentials: An ambiguity associated with analysis of variance models. Electroencephalography and Clinical Neurophysiology, 62, 203-208.

Meuter, R. F. I., \& Allport, A. (1999). Bilingual language switching in naming: Asymmetrical costs of language selection. Journal of Memory and Language, 40, 25-40.

Münte, T. F., Heinze, H. J., \& Mangun, G. R. (1993). Dissociation of brain activity related to syntactic and semantic aspects of language. Journal of Cognitive Neuroscience, 3, 335-344.

Münte, T. F., Heinze, H- J., Matzke, M., Wieringa, B. M., \& Johannes, S. (1998). Brain potentials and syntactic violations revisited: No evidence for specificity of the syntactic positive shift. Neuropsychologia, 36, 217-226.

Neville, H. J., Nicol, J. L., Barss, A., Forster, K. I., \& Garrett, M. F. (1991). Syntactically based sentence processing classes: Evidence from event-related brain potentials. Journal of Cognitive Neuroscience, 3, 151-165.

Oldfield, R. C. (1971). The assessment and analysis of handedness: The Edinburgh inventory. Neuropsychologia, 9, 97-113.

Osterhout, L., Bersick, M., \& McLaughlin, J. (1997). Brain potentials reflect violations of gender stereotypes. Memory and Cognition, 25, 273-285.

Osterhout, L., \& Holcomb, P. J. (1992). Event-related brain potentials elicited by syntactic anomaly. Journal of Memory and Language, 31, 785-806.

Paller, K. A., \& Kutas, M. (1992). Brain potentials during memory retrieval provide neurophysiological support for the distinction between conscious recollection and priming. Journal of Cognitive Neuroscience, 4, 375-391.

Pavlenko, A. (1999). New approaches to concepts in bilingual memory. Bilingualism: Language and Cognition, 2, 209-226.

Picton, T. W. (1992). The P300 wave of the human event-related potential. Journal of Clinical Neurophysiology, 9, 456-479.

Price, C. J., Green, D. W., \& von Studnitz, R. (1999). A functional imaging study of translation and language switching. Brain, 122, 2221-2235.

Ranganath, C., \& Paller, K. A. (1999). Frontal brain activity during episodic and semantic retrieval: Insights from event-related potentials. Journal of Cognitive Neuroscience, 11, 598-609.

Rugg, M. D., Allan, K., \& Birch, C. S. (2000). Electrophysiological evidence for the modulation of retrieval orientation by depth of study processing. Journal of Cognitive Neuroscience, 12, 664678.

Senkfor, A. J., \& Van Petten, C. (1998). Who said what? An event-related potential investigation of source and item memory. Journal of Experimental Psychology: Learning, Memory, and Cognition, 24, 1005-1025.

Sohn, M-H., Ursu, S., Anderson, J. R., Stenger, V. A., \& Carter, C. S. (2000). The role of prefrontal cortex and posterior parietal cortex in task switching. Proceedings of the National Academy of Sciences of the United States of America, 97, 13448-13453. 
Sommer, W., Leuthold, H., \& Matt, J. (1998). The expectancies that govern the P300 amplitude are mostly automatic and unconscious. Behavioral and Brain Sciences, 21, 149-168.

Sridhar, S. N., \& Sridhar, K. K. (1980). The syntax and psycholinguistics of bilingual code mixing. Canadian Journal of Psychology, 34, 407-416.

Taylor, W. L. (1953). “Cloze procedure', A new tool for measuring readability. Journalism Quarterly, 30, 415-433.

Thomas, M. S. C., \& Allport, A. (2000). Language switching costs in bilingual visual word recognition. Journal of Memory and Language, 43, 44-66.

Van Petten, C., Senkfor, A. J., \& Newberg, W. M. (2000). Memory for drawings in locations: Spatial source memory and event-related potentials. Psychophysiology, 37, 551-564.

Weber-Fox, C. M., \& Neville, H. J. (1996). Maturational constraints on functional specializations for language processing: ERP and behavioral evidence in bilingual speakers. Journal of Cognitive Neuroscience, $\mathbf{8}, 231-256$. 